Noname manuscript No.

(will be inserted by the editor)

\title{
A length scale defining partially-resolved boundary-layer turbulence simulations
}

\author{
Robert J. Beare
}

the date of receipt and acceptance should be inserted later

\begin{abstract}
Numerical weather prediction (NWP) model forecasts at horizontal grid lengths in the range of $100 \mathrm{~m}$ to $1 \mathrm{~km}$ are now possible. Within this range of grid lengths, the convective boundary layer is partially resolved and thus in the socalled 'grey zone'. For simulations in the grey zone, numerical dissipation sources from both the advection scheme and the subgrid model are likely to be significant. Until now, these effects have not been incorporated fully into our understanding of the grey zone. In order to quantify these effects, a dissipation length scale is defined based on the second moment of the turbulent kinetic energy (TKE) spectrum.

An ensemble of simulations of a convective boundary layer are performed using a large-eddy model across the grey-zone resolutions and for a range of subgrid model, advection scheme and vertical grid configurations. The dissipation length scale distinguishes the effects of the different model configurations in the grey zone. In the middle of the boundary layer, the resolved TKE is strongly controlled by the numerical dissipation. This leads to a similarity law for the resolved TKE in the grey zone using the dissipation length scale. A new definition of the grey zone emerges where the inversion depth and dissipation length scale are the same size. This contrasts with the typical definition using the horizontal grid length. At the inversion, however, the variation of the dissipation length scale with grid length is less predictable, reflecting significant challenges for modelling entrainment in the grey zone. The dissipation length scale is thus a simple diagnostic to aid both NWP and large-eddy modellers in understanding the grey zone.
\end{abstract}

Keywords Grey zone; Boundary layer; Subgrid model; Advection scheme; Power spectrum; Dissipation

\section{Introduction}

During the diurnal cycle, the typical length scale of boundary-layer eddies varies between of order $1 \mathrm{~km}$ by day (convective and neutral boundary layers) to much smaller values at night (stable boundary layers). Until recently, numerical weather

College of Engineering, Mathematics and Physical Sciences, University of Exeter, EX4 4QF. Email: r.j.beare@ex.ac.uk 
prediction (NWP) models used a horizontal grid length $(\Delta x)$ much larger than the size of the boundary-layer eddies. The scale separation meant that columnbased parametrizations were formally justified. However, with current computing power, and in order to provide more skillful regional forecasts, operational weather centres now run limited area models at horizontal grid lengths as small as a few kilometres. For example, the 1.5-km grid length configuration of the Met Office Unified Model provides high-resolution forecasts of severe precipitation and fog for domains covering the UK (Lean and Browning 2012).

For small horizontal and vertical grid lengths $(25 \mathrm{~m}$ or smaller $)$, the eddies of the convective boundary layer (CBL) are well resolved (Sullivan and Patton 2011). Such simulations are in the large-eddy simulation (LES) regime. However, at larger grid lengths that are not so large that the eddies are totally unresolved, the simulation is then partially resolved. At these resolutions the turbulence is neither fully represented by a vertical column scheme, as vertical motions are still permitted, nor can it be considered a full LES, as the turbulence is only partially resolved. Such a regime was termed the 'terra-incognita' by Wyngaard (2004) and is also commonly referred to as the 'grey zone'. For several years, the grey zone has been an issue for NWP of convective systems (Craig and Dörnbrack 2008). The grey zone is now also a pressing practical issue for NWP of the atmospheric boundary layer.

The grey zone is often defined as when $\Delta x$ and the energy-containing scales are the same size (Wyngaard 2004). However, numerical dissipation sources of the model can typically act over several $\Delta x$. Whilst subgrid models are often formulated as a diffusion operator, advection schemes can also have implicit diffusion due to truncation errors (errors due to finite resolution, Chow and Moin 2003). Since the truncation errors are at the same scale as the boundary-layer eddies in the grey zone, the implicit diffusion from the advection scheme could be as significant as the subgrid model. However, the role of the advection scheme in the boundary-layer grey zone has so far been overlooked. In addition to dissipative effects, the truncation can also give dispersion errors. The idea of implicit diffusion from the truncation error of the advection scheme is an established idea in computational fluid dynamics. The modified equation analysis of Warming and Hyett (1974) demonstrated that the effective equation that is being solved has extra terms, some of which behave as increased diffusion. The aim of this study is to define a scale, the dissipation length scale, which encasulates the effects of these numerical dissipation sources.

There are still relatively few studies modelling the boundary-layer grey zone, notably that of Honnert et al. (2011) for convective and cumulus boundary layers and Dorrestijn et al. (2013) for the cumulus boundary layer. Starting with a high-resolution LES, Honnert et al. (2011) filtered the model fields onto coarser horizontal grid lengths. They then evaluated the subgrid term as the grid-length changes from the LES regime into the grey zone. Honnert et al. (2011) did this for clear and cloudy boundary-layer cases and formulated similarity functions for the ratio of subgrid to total fluxes in terms of the variable $\Delta x /\left(h+h_{c}\right)$ where $h$ and $h_{c}$ are the depths of the boundary layer and cloud layers respectively. A strength of their approach was showing the different functional variations that apply for the variables: TKE, potential temperature and moisture mixing ratio. However, their results applied for a single model configuration. There thus remains the question of how to account for the variations due to different numerical model configurations. 
These variations can be summarized by the subgrid models and advection schemes used. Here, we formulate a length scale, based on the second moment of the TKE power spectrum, with the aim of separating the effects of different subgrid models and advection schemes.

\section{Method}

Figure 1 illustrates our approach of using the TKE power spectrum $\left(S_{e}\right)$ to define the grey zone. From that spectrum we identify three key length scales. The peak of the spectrum is given by $l_{p}$ and is typically controlled by the largest eddies of the turbulence, for example the boundary-layer depth. For a horizontal wavenumber $(k)$ above that of the largest eddies, the ideal spectrum follows the inertial subrange and varies as the well-established $k^{-5 / 3}$ Kolmogorov law (Davidson 2004). Above a certain wavenumber, the spectrum of the simulation will typically have more dissipation than that associated with the Kolmogorov law. In the vicinity of the dissipation scale $\left(l_{d}\right)$, above wavenumber $2 \pi / l_{d}$, the simulation's spectrum deviates increasingly from the ideal $k^{-5 / 3}$ spectrum. Note that $l_{d}$ does not need to lie at the turning point from the ideal spectrum as in the schematic; however it does need to change in response to the different numerical dissipation values. Finally, the spectrum terminates in the vicinity of the filter width $\left(\Delta_{f}\right)$,

$$
\Delta_{f}=(\Delta x \Delta y \Delta z)^{\frac{1}{3}}
$$

where $\Delta x, \Delta y$ and $\Delta z$ are the grid lengths in the $x, y$ and $z$ Cartesian-coordinate directions respectively. The key feature is that $l_{d}>\Delta_{f}$, encapsulating the fact that numerical dissipation can act over several grid lengths. In Fig. 1a, the scenario for LES is illustrated. Here the peak $\left(l_{p}\right)$ and the dissipation $\left(l_{d}\right)$ length scales are clearly separated and there is a well-defined inertial sub-range. However, at a sufficiently coarse grid length, the peak and dissipation length scales become similar in size and the grey zone results, defined as

$$
l_{p} \sim l_{d}
$$

Since $l_{d}>\Delta_{f}$, the grey zone definition in Eq. 2 occurs at smaller grid lengths than the definition $l_{p} \sim \Delta_{f}$. Unlike the LES regime, the numerical dissipation has a strong influence on the peak of the spectrum in the grey zone.

\subsection{Dissipation length scale}

The more numerically dissipative a model is, the less resolved the turbulence will be for a given horizontal grid length. In order to quantify this effect, we now use the TKE spectrum to calculate a length scale representing the numerical dissipation. The dissipation associated with an infinite spectrum with molecular viscocity, $\nu$, is

$$
\epsilon=2 \nu \int_{0}^{\infty} k^{2} S_{e}(k) d k
$$


and the scale selectivity of the dissipation is determined by the integral of $k^{2}$ over $S_{e}$. Using this insight, we define a dissipation wavenumber $\left(k_{d}\right)$ for a finite spectrum that also scales with this integral

$$
k_{d}^{2}=\frac{\int_{k_{0}}^{k_{1}} k^{2} S_{e}(k) d k}{\int_{k_{0}}^{k_{1}} S_{e}(k) d k},
$$

where $k_{0}$ and $k_{1}$ define the lower and upper wavenumber limits of the spectrum. The dissipation length scale is then

$$
l_{d}=\frac{2 \pi}{k_{d}} .
$$

Length scales could also be defined based on other moments of the TKE spectrum, with higher moments favouring smaller scales, and lower moments larger scales. However, none of these is linked in the same way as Eqs. 4 and 5 with the functional form of the dissipation.

The dissipation length scale can now potentially separate the relative effects of numerical dissipation in different model configurations. For example, for a given grid length, a more dissipative simulation will have a larger value of $l_{d}$. It is important to note that this dissipation length scale is due to the TKE spectrum associated with the simulation, including implicit damping from the advection scheme, and not just the subgrid viscosity.

\subsection{A diagnostic of the spectrum}

The dissipation length scale diagnoses the behaviour of the TKE spectrum with different model configurations in the grey zone. In cases where the TKE spectrum follows the 'typical' form in Fig. 1, with the spectrum maintaining its shape and always falling below the Kolmogorov law at high wavenumbers, the dissipation length scale will increase with both the horizontal grid length and the numerical dissipation. However, if the shape of the spectrum changes significantly with increasing horizontal grid length, for example sometimes lying above the Kolmogorov law at high wavenumbers due to effects other than numerical dissipation, then the dissipation length scale will not change as predictably with horizontal grid length.

\subsection{Grey-zone similarity variable}

The independent similarity variable for the grey zone should define the degree of resolution of the turbulence. The ratio $z_{i} / \Delta x$, where $z_{i}$ is the inversion depth, was used by Honnert et al. (2011) to parametrize the subgrid turbulence in the grey zone. The inversion depth is equivalent to the boundary-layer depth for a CBL. This is a natural first choice as large values of $z_{i} / \Delta x$ correspond to a well resolved simulation. However, if we wish to include different model configurations, the ratio $z_{i} / l_{d}$ may be more appropriate. In simulations where the TKE is strongly

controlled by the numerical dissipation in the grey zone, we anticipate a set of similarity functions, $g_{n}$,

$$
\bar{e}_{r e s}\left(z_{n}\right)=g_{n}\left(\frac{z_{i}}{l_{d}}\right) \text {, }
$$


where $\bar{e}_{\text {res }}$ is the resolved area/time-averaged TKE at height $z_{n}$. It is important to note that the similarity functions for the resolved flow in Eq. 6 are valid within the grey zone $\left(z_{i} \sim l_{d}\right)$, but not in the LES regime.

\subsection{Convective boundary-layer case}

LES models have been used for many years as a basis for informing parametrization in NWP models. In grey-zone research, the LES provides a robust, well-tested highresolution reference for convective and other types of boundary layer. Since NWP models have not yet tested such a limit, this makes LES a preferable starting point for studying the grey zone. A potential drawback of the LES in the grey zone is that the subgrid model does not become a typical single-column NWP boundary-layer parametrization scheme in the coarse resolution limit. Therefore, the switching point of an LES model between un-resolved motions and the grey zone may differ in detail from NWP models. However, as with Honnert et al. (2011), we view that useful diagnostics and ideas can be developed within the simpler LES environment and then be applied to more complicated NWP models.

As the resolution of NWP models increases, it is the CBL, with its depth of order $1 \mathrm{~km}$, which is the first section of the diurnal cycle to enter the grey zone. We thus chose a standard CBL case that has been thoroughly studied in the literature forced by a weak geostrophic wind and strong surface heat flux. It has been used by many others in the past and was recently simulated at extremely high resolution by Sullivan and Patton (2011). The Met Office large-eddy model (Mason and Derbyshire 1990) is used to simulate a version of the Sullivan and Patton (2011) case adapted for the grey-zone problem.

Full details of the case are given by Sullivan and Patton (2011), so here we summarize the set-up. The initial potential temperature profile is a mixed layer up to an inversion height with a strong overlying stratification. The surface sensible heat flux is constant at $241 \mathrm{Wm}^{-2}$. The initial inversion depth is slightly modified from that used by Sullivan and Patton (2011), using $1 \mathrm{~km}$ instead of $974 \mathrm{~m}$. This is to give an integer number of the vertical grid lengths within the inversion depth for the coarser resolution simulations. The peak of the TKE power spectrum is set as: $l_{p}=1.9 z_{i}$.

\subsection{Grey-zone experiments}

Simulations were performed for each model configuration at horizontal grid lengths of $25,50,100,200$ and $400 \mathrm{~m}$. The horizontal grid length of $25 \mathrm{~m}$ is near the limit of the converged LES defined by Sullivan and Patton (2011), whilst the horizontal grid length of $400 \mathrm{~m}$ is firmly in the grey zone. Simulations were also performed at a 800-m horizontal grid length, but these will not be shown as most had negligible resolved turbulence; this is also an indication that using the criterion of the horizontal grid length equalling the inversion depth $(1 \mathrm{~km})$ for the grey zone is not accurate. The grey zone applies for much smaller grid lengths than $1 \mathrm{~km}$. To give a sufficient number of grid points in the horizontal for the grey-zone grid lengths, we used a horizontal domain length that was approximately double that used by Sullivan and Patton (2011). A domain of $9.6 \mathrm{~km} \times 9.6 \mathrm{~km}$ in the horizontal 


\begin{tabular}{lccc}
\hline Simulation & Adv. mom. & Adv. $\theta$ & $C_{s}$ \\
\hline Control simulation & P-W & TVD & 0.23 \\
TVD on $(u, v, w)$ experiment & TVD & TVD & 0.23 \\
P-W on $\theta$ experiment & P-W & P-W & 0.23 \\
$C_{s}=0.207$ experiment & P-W & TVD & $\mathbf{0 . 2 0 7}$ \\
$C_{s}=0.115$ experiment & P-W & TVD & $\mathbf{0 . 1 1 5}$ \\
$C_{s}=0.46$ experiment & P-W & TVD & $\mathbf{0 . 4 6}$ \\
$\Delta \mathbf{z}=\mathbf{4 0 ~} \mathbf{m}$ experiment & P-W & TVD & 0.23 \\
$\Delta \mathbf{z}=\mathbf{8 0} \mathbf{m}$ experiment & P-W & TVD & 0.23 \\
\hline
\end{tabular}

Table 1 A summary of the simulations. Changes relative to the control simulation marked in bold. Total Variation Diminishing (TVD) and Piacsek-Williams (P-W) are advection scheme options for either momentum (Adv. mom.) or potential temperature (Adv. $\theta$ ). $C_{s}$ is the Smagorinsky constant. The $\Delta z=40 \mathrm{~m}, \Delta z=80 \mathrm{~m}$ experiments are the constant vertical grid-length configurations.

and $2 \mathrm{~km}$ in the vertical was used for all simulations apart from the $25-\mathrm{m}$ horizontal grid length where a $4.8 \mathrm{~km} \times 4.8 \mathrm{~km}$ horizontal domain was sufficient. For many of the simulations, the vertical grid length was set to $\Delta z=0.4 \Delta x$ to give a vertical resolution of the convective turbulence that was consistent with the horizontal grid length. However, it is also interesting to consider the constant vertical grid length case as this is often used in NWP. Experiments with constant vertical grid lengths, $\Delta z=40 \mathrm{~m}$ and $\Delta z=80 \mathrm{~m}$, were used here. The simulations were run for 4 hours. The fluxes, variances and potential temperature profiles were averaged over the horizontal domain and, as in Sullivan and Patton (2011), over 15 eddy overturning times for statistical robustness, from 1.5 to $3.5 \mathrm{hr}$.

Table 1 summarizes the simulations that include different subgrid model, advection scheme and vertical grid settings and are simulated at all the resolutions previously defined. Whilst there is a large range of possible subgrid model options in LES (including dynamic and stochastic backscatter), the aim of this study was to quantify the compensating role of the advection scheme for a given subgrid model. We thus confined ourselves to the Smagorinsky model which allows a simple modification of the subgrid dissipation by varying the Smagorinsky constant $\left(C_{s}\right)$. The mixing length for the Smagorinsky model in the Met Office LES model scales as $C_{s} \Delta x$ in the domain interior. The configuration of the control simulation is the default used in the Met Office LES model, with a Smagorinsky constant of 0.23. In order to examine the role of the dissipation of the subgrid model, other experiments consisted of a doubling $\left(C_{s}=0.46\right)$, halving $\left(C_{s}=0.115\right)$ and $10 \%$ reduction $\left(C_{s}=0.207\right)$ of the Smagorinsky constant. For advection, the options of a centred-difference advection scheme (Piacsek and Williams 1970, P-W) as well as a Total Variation Diminishing scheme (Leonard et al. 1993, TVD) were used. Since the P-W scheme is not monotone, it is less dissipative than the TVD scheme so changing between the TVD and P-W on momentum or potential temperature provides a way of changing the implicit diffusion of the advection scheme. Thus, typically the TVD on $(u, v, w)$ experiment will be more dissipative than the control simulation, whilst the $\mathrm{P}-\mathrm{W}$ on $\theta$ experiment will be less dissipative. 


\section{Results}

We first set the scene by describing the grey-zone CBL simulations using the usual diagnostics. We then show the TKE spectra and diagnosed dissipation length scales.

\subsection{CBL simulations in the grey zone}

Figure 2 shows the vertical velocity at height $500 \mathrm{~m}$ (approximately half of the boundary-layer depth) for horizontal grid lengths from $400 \mathrm{~m}$ down to $50 \mathrm{~m}$, successively halving the grid length. At 50-m grid length the simulations are close to the LES regime. The updrafts at 400-m grid length are both broader horizontally and smaller in amplitude compared with the LES limit at $50 \mathrm{~m}$. As the grid length decreases from $400 \mathrm{~m}$ to $50 \mathrm{~m}$, the updrafts narrow and increase in magnitude; moreover, the edges of the updrafts develop increasing turbulent fine structure. Whilst the 50-m grid length simulation is visibly a turbulent simulation, the partial resolution of the fields at 400-m grid length means that the LES is compromised and exists in the grey zone. In order to provide a converged bench mark, we also performed a 25-m horizontal grid length simulation, but this time with half the domain length, the same domain size as Sullivan and Patton (2011).

Figure 3 shows vertical profiles of time-area averaged potential temperature and buoyancy flux for the control simulation. For the potential temperature (Fig. $3 \mathrm{a}$ ), there is the least variation between the resolutions in the middle of the boundary layer $(\approx 0.1 \mathrm{~K})$, moderate variations near the boundary layer top and bottom $(\approx 0.5 \mathrm{~K})$ and large variations above the boundary layer top $(\approx 4 \mathrm{~K})$. These variations are explained by the total (resolved plus subgrid) buoyancy flux (Fig. 3b), which is prescribed at the surface, has small variations in the middle of the boundary layer, and very significant variations at the inversion. The insensitivity of the total flux in the middle of the boundary layer is despite the reduction of resolved flow seen previously in Fig. 2. This is due to the subgrid model's flux compensating for the changes in resolved flux. Figure 4 shows the variation with horizontal grid length of the subgrid and resolved components of buoyancy flux in the middle of the boundary layer. As the horizontal grid length increases, the resolved flux decreases, but the subgrid flux increases to compensate for this. The LES thus has a degree of 'scale-awareness' in its formulation. By a horizontal grid length of $400 \mathrm{~m}$, the subgrid flux has become a significant proportion (46\%) of the resolved flux, confirming that the simulation is in the grey zone at this height.

At the inversion, however, the total buoyancy flux is not constant with resolution (Fig. 3). As the horizontal grid length is decreased, the entrainment flux decreases, although not monotonically, to the converged value at $25 \mathrm{~m}$, in agreement with Sullivan and Patton (2011). Whilst the total flux is relatively insensitive to resolution in the middle of the boundary layer, the resolved fields are likely to be more sensitive. It is the resolved fields that define whether the simulations are in the grey zone and are the focus of of our study.

Figure 5 shows the variation of the vertical velocity variances with resolution for the control simulation and the TVD on $(u, v, w)$ experiment. At a horizontal grid length of $25 \mathrm{~m}$, there is negligible difference between the maxima and shapes of the vertical velocity variance profiles. However, as the horizontal grid length coarsens 
to $400 \mathrm{~m}$, significant differences emerge. Compared with the control simulation (Fig. 5a), the TVD on $(u, v, w)$ experiment (Fig. 5b) reduces the vertical velocity variance near the surface for a given horizontal grid length. When a horizontal grid length of $400 \mathrm{~m}$ is reached, there is little resolved vertical velocity variance near the surface in the TVD on $(u, v, w)$ experiment and the simulation is effectively poorly resolved in this region. There is also more significant damping at the inversion in the TVD on $(u, v, w)$ experiment compared with the control simulation. The TVD on $(u, v, w)$ experiment thus exhibits more implicit dissipation than the control simulation due to the monotone advection scheme.

Figure 6 is the equivalent comparison of Fig. 5, but for the resolved TKE. In a similar way to the vertical velocity variance, the resolved TKE decreases monotonically with increasing $\Delta x$ in the middle of the boundary layer, and more rapidly in the TVD on $(u, v, w)$ experiments. However, at the inversion, the behaviour with resolution is not as predictable, with the TKE initially decreasing with increasing $\Delta x$, but then increasing in both the control simulation and TVD on $(u, v, w)$ experiment at the largest $\Delta x$. We will return to the less predictable behaviour at the inversion compared with the middle of the boundary layer in Section 3.4.

\subsection{Spectra and dissipation length scales}

Central to our analysis of the grey zone is the TKE power spectrum. Figure 7 shows one-dimensional TKE power spectra plotted against horizontal wavenumber at the height of the middle of the boundary layer. Figure 7 compares the spectra for the control simulation and TVD on $(u, v, w)$ experiment. The TVD on $(u, v, w)$ experiment (Fig. 7b) falls off more significantly from the ideal Kolmogorov law $\left(k^{-5 / 3}\right)$ than the control simulation (Fig. 7a) for horizontal grid lengths between $50 \mathrm{~m}$ and $400 \mathrm{~m}$. This is due to the greater implicit diffusion of the TVD scheme. At horizontal grid lengths of $200 \mathrm{~m}$ and $400 \mathrm{~m}$, for both the control simulation and the TVD on $(u, v, w)$ experiment, the wavenumber for fall-off from the ideal Kolmogorov law is now in the region of the peak wavenumber $k l_{p} / 2 \pi \approx 1$. The simulations are now in the grey zone (see Fig. 1).

The dissipation length scale $\left(l_{d}\right.$, Eqs. 4 and 5$)$ quantifies the different falloffs from the ideal spectrum shown previously. As it is the integral of $k^{2}$ over the spectrum, $l_{d}$ emphasises the higher wavenumber scales. The wavenumber of $l_{d}$ for each horizontal grid length $l_{p} / l_{d}$ is marked as a vertical line at the top of Figs. $7 \mathrm{a}$ and b. Although the wavenumbers of the dissipation length scales don't align exactly with the point of fall-off from the Kolmogorov law, they do scale proportionately to it. For a given $\Delta x$, when the model numerics are more dissipative, as in Fig. 7b, then the fall-off from the Kolmogorov law is at a smaller wavenumber, $1 / l_{d}$ is also smaller and thus $l_{d}$ is larger.

Figure 8 shows $l_{d}$ for the middle of the boundary layer plotted against $\Delta x$. Since NWP modellers often use the horizontal grid length instead of the filter scale when describing the grey zone, we use it here in presenting our results. The values of $l_{d}$ separate with increasing $\Delta x$, showing how the simulations are increasingly sensitive to model configuration when in the partially-resolved grey zone. The values of $l_{d}$ are also several $\Delta x$. For example, at $\Delta x=100 \mathrm{~m}, l_{d} \sim 13 \Delta x$ for the TVD on $(u, v, w)$ experiment but $l_{d} \sim 9 \Delta x$ for the control simulation. The curves separate according to the known dissipation of the model configurations. Both the TVD on 
$(u, v, w)$ and the double Smagorinsky constant $\left(C_{s}=0.46\right)$ experiments have larger dissipation length scales than the control simulation. The P-W on $\theta$ experiment has a slightly smaller dissipation length scale than the control simulation. The values of $l_{d}$ are smaller proportionately for the $C_{s}=0.207$ and $C_{s}=0.115$ configurations. The values of $l_{d}$ in the middle of the boundary layer are relatively insensitive to the choice of vertical grid configuration. The dissipation length scale thus encapsulates the sensitivity of the TKE spectrum to both horizontal grid length and model configuration.

\subsection{Parametrization of resolved TKE}

Figure 9a shows the resolved TKE in the middle of the boundary layer as a function of $z_{i} / \Delta x$. For all curves there is a significant monotonic decrease at coarse resolutions (decreasing $z_{i} / \Delta x$ ), showing the transition from LES (right of figure) to the grey zone (left of figure). However, the rate of transition between LES and the grey zone depends on the model configuration. For example, the $C_{s}=0.46$ curve falls off more rapidly than control.

In contrast, Fig. $9 \mathrm{~b}$ shows the resolved TKE in the middle of the boundary layer as a function of $z_{i} / l_{d}$. Here the coarse resolution curves (left of figure) collapse onto a common curve. At higher resolutions, the resolved TKE is less controlled by the numerical dissipation as the simulations are in the LES regime. The curves at coarse resolution approximate the following simple similarity function (grey line in Fig. 9b):

$$
\bar{e}_{r e s}^{m}=\bar{e}_{h}^{m} \tanh \left(\frac{z_{i}}{l_{d}}\right),
$$

where $\bar{e}_{r e s}^{m}$ is the resolved TKE in the middle of the boundary layer and $\bar{e}_{h}^{m}$ is the high-resolution value. The grey zone can now be defined as when the curves collapse onto one another (Fig. 9b) at approximately $z_{i} / l_{d}<0.7$, as it is here that the simulations are influenced by the numerical dissipation of the model. This now shows that the onset of the grey zone will be dependent on the inherent dissipation in the model and use of the similarity variable $z_{i} / l_{d}$ is more robust than $z_{i} / \Delta x$.

\subsection{Inversion behaviour}

The grey-zone behaviour in the middle of the boundary layer, where the resolved TKE monotonically decreases with increasing $\Delta x$, contrasts with that at the inversion where the resolved TKE changes more erratically with grid length (Fig. 6a). The TKE spectrum at the inversion for the control simulation (Fig. 10a) also shows a different behaviour from the middle of the boundary layer for resolutions in the grey zone. The peak in the energy spectrum actually increases at coarser horizontal grid lengths (Fig. 10a) and the high wavenumber section of the spectrum lies above the $k^{-5 / 3}$ line. It is also somewhat surprising that, compared with the middle of the boundary layer, the control simulation follows the $k^{-5 / 3}$ line so closely at the inversion at high resolutions. One aspect of these differences is that the inversion is a stratified layer, whereas the middle of the boundary layer is unstratified. The effect of enhanced numerical dissipation is still clearly present in the TVD on $(u, v, w)$ experiment (Fig. 10b) with the high wavenumbers falling 
below the $k^{-5 / 3}$ line. The spectrum of the TVD on $(u, v, w)$ experiment at the inversion follows the 'typical' variation with $\Delta x$ as schematised in Fig. 1, whilst the control simulation does not.

These differences are also shown in the variation of $l_{d}$ with $\Delta x$ at the inversion (Fig. 11). The $l_{d}$ curves do not vary in such a systematic way as the middle of the boundary layer, with some curves crossing the others at coarser horizontal grid lengths. The variation of $l_{d}$ does not always follow the changes in model numerical dissipation as in the middle of the boundary layer. The TVD on $(u, v, w)$ experiment still has a larger dissipation scale than the control simulation. This is consistent with the enhanced dissipation provided by the TVD scheme. However the $\mathrm{P}-\mathrm{W}$ on $\theta$ experiment, with smaller numerical dissipation than the control simulation, now has larger values of $l_{d}$ than the control simulation, demonstrating a complicated interaction between the advection scheme and the inversion. The values of $l_{d}$ at the inversion are also more sensitive to the choice of vertical grid than in the middle of the boundary layer, with the constant vertical grid length experiment, $\Delta z=40 \mathrm{~m}$, giving values more separated from the control simulation. This is consistent with the greater sensitivity to vertical resolution at the inversion.

These complications prevent formation of simple similarity functions for the resolved TKE with $z_{i} / l_{d}$ at the inversion. The complexity of the sensitivity of entrainment in the grey zone to model configuration is perhaps unsurprising given the challenges modelling entrainment described previously by other authors (e.g. Bretherton et al. 1999).

\section{Conclusions}

In this paper, we defined a characteristic length scale for the atmospheric boundary layer in the grey zone. The dissipation length scale $\left(l_{d}\right)$ was based on the second moment of the turbulent kinetic energy spectrum. For the middle of a convective boundary layer it accounted for both variations in the advection scheme and subgrid model. Such variations have not been fully accounted for before in the boundary-layer grey zone. The definition of key length scales for the resolution problem was previously pursued by Craig and Dörnbrack (2008) for moist convection. They defined a buoyancy length scale and initial thermal diameter, and showed that the smaller of these needed to be resolved.

Previously, Honnert et al. (2011) proposed the ratio of the inversion depth to horizontal grid length $\left(z_{i} / \Delta x\right)$ as a similarity variable for the grey zone. Using this variable, they showed a reasonable collapse onto a similarity function for the subgrid turbulence. However, this was for only one model configuration. We have showed that this similarity variable does not fully account for variations in the dissipation that arise from differences in either subgrid model or advection scheme. When the similarity variable $z_{i} / l_{d}$ was used, the resolved TKE in the middle of the boundary layer collapsed onto a common curve for approximately $z_{i} / l_{d}<0.7$. This formed a new definition for the grey zone that is more robust than the often used $z_{i} \sim \Delta x$ (Wyngaard 2004). The value of $l_{d}$ is typically several $\Delta x$, so the $z_{i} / l_{d}<0.7$ criterion means that the grey zone can occur at horizontal grid lengths smaller than the inversion depth. At the inversion, the variation of dissipation length scale with model configuration was more complex, preventing an equivalent similarity law. This reflects other authors' experience of modelling entrainment (e.g. Bretherton 
et al. 1999), and it is also analogous to the problem of resolution in the surface layer. The new diagnostic has thus highlighted examples where the variations of model configurations are relatively easy to understand (such as the middle of the CBL) and those where it is complex (such as the inversion). In either case, the dissipation length scale has proved useful in classifying these behaviours and, even in the complex cases, provides a reference point for further studies of their parametrization.

The dissipation length scale could be used in NWP models to distinguish model configurations in the grey zone. Also, the similarity function in Eq. 7 could form the basis of a NWP scheme where the single-column boundary-layer scheme is partially turned off and the Smagorinsky model partially turned on in the grey zone. Moreover, LES modellers want to avoid the grey zone, and the new diagnostic provides a way of checking that this is the case. Given the potential for model dependence in the grey zone, it is informally considered a 'messy' area of research with difficulties defining the key scales rigorously. Here we have provided a simple but rigorous length scale definition for the grey zone that could form the basis for other diagnostics. Future work could also extend the application of the dissipation length scale to the neutral and stable boundary layer.

\section{Acknowledgement}

I am grateful for discussions with Dr David Thomson, Met Office UK, concerning the formulation of the dissipation length scale. The computations reported here were performed using the University of Exeter Supercomputer.

\section{References}

Bretherton, C. S., M. K. MacVean, P. Bechtold, A. Chlond, W. R. Cotton, J. Cuxart, H. Cuijpers, M. Khairoutdinov, B. Kosovic, D. Lewellen, C. H. Moeng, P. Siebesma, B. Stevens, D. E. Stevens, I. Sykes, and M. C. Wyant. 1999. An intercomparison of radiatively driven entrainment and turbulence in a smoke cloud as simulated by different numerical models. Q. J. R. Meteorol. Soc. 125: 391-423.

Chow, F. K., and P. Moin. 2003. A further study of numerical errors in large-eddy simulations. J. Comput. Phys. 184: 366-380.

Craig, G. C., and A. Dörnbrack. 2008. Entrainment in cumulus clouds: what resolution is cloud-resolving? J. Atmos. Sci. 65: 3978-3988.

Davidson, P. A. 2004. Turbulence. Oxford University Press, Oxford. 678 pp.

Dorrestijn, J., D. T. Crommelin, A. P. Siebesma, and H. J. J. Jonker. 2013. Stochastic parameterization of shallow cumulus convection estimated from high-resolution model data. Theor. Comput. Fluid Dyn. 27: 133-148.

Honnert, R., V. Masson, and F. Couvreux. 2011. A diagnostic for evaluating the representation of turbulence in atmospheric models at the kilometric scale. J. Atmos. Sci. 68: 3112-3131.

Lean, H. W., and K. A. Browning. 2012. Quantification of the importance of wind drift to the surface distribution of orographic rain on the occasion of the extreme Cockermouth flood in Cumbria. Q. J. R. Meteorol. Soc. 139: 1342-1353.

Leonard, B. P., M. K. MacVean, and A. P. Lock. 1993. Positivity-preserving numerical schemes for multidimensional advection. NASA Technical Memorandum 106055 (ICOMP-93905), $62 p p$.

Mason, P. J., and S. H. Derbyshire. 1990. Large-eddy simulation of the stably-stratified atmospheric boundary layer. Boundary-Layer Meteorol. 53: 117-162.

Piacsek, S. A., and G. P. Williams. 1970. Conservation properties of convection difference schemes. J. Comput. Phys. 6: 392-405. 
Sullivan, P. P., and E. G. Patton. 2011. The effect of mesh resolution on convective boundary layer statistics and structures generated by large-eddy simulation. J. Atmos. Sci. 68: 23952415.

Warming, R. F., and B. J. Hyett. 1974. The modified equation approach to the stability and accuracy analysis of finite-difference methods. J. Comp. Phys. 14: 159-179.

Wyngaard, J. C. 2004. Toward numerical modeling in the "Terra Incognita". J. Atmos. Sci. 61: $1816-1825$. 

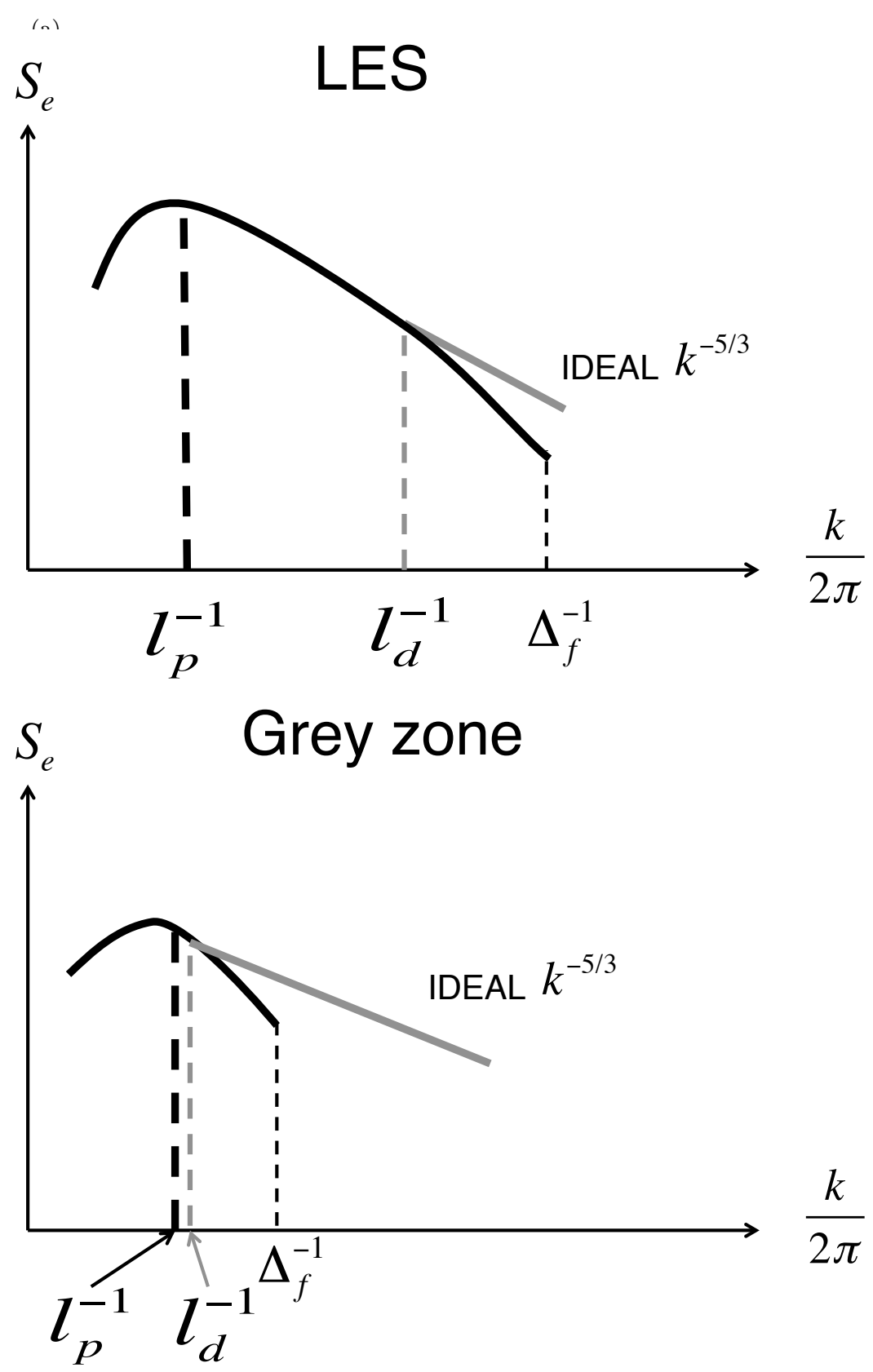

Fig. 1 A schematic showing TKE spectrum $\left(S_{e}\right)$ against horizontal wavenumber $(k)$ for a simulation (black line) and an ideal $k^{-5 / 3}$ law (grey line). The length-scales annotated are: the spectral peak $\left(l_{p}\right)$, the dissipation length scale $\left(l_{d}\right)$ and the filter width $\left(\Delta_{f}\right)$. Illustrations of these are given for: (a) a large-eddy simulation (LES), (b) a simulation in the grey zone. 
(a)

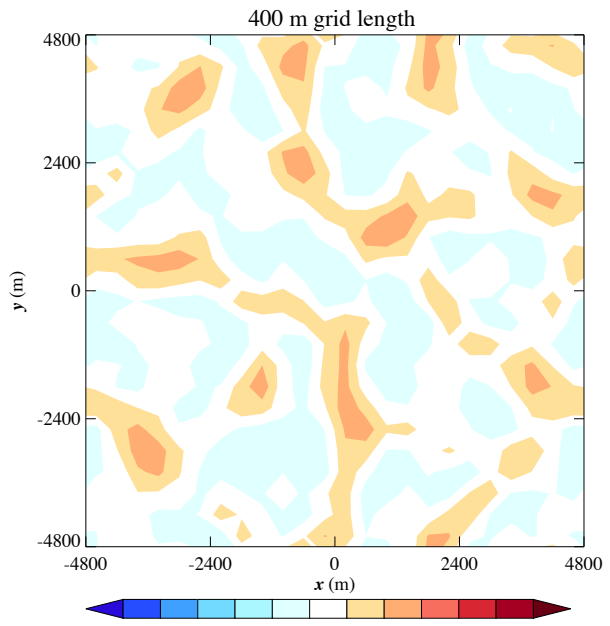

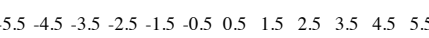

Vertical velocity $\left(\mathrm{m} \mathrm{s}^{-1}\right)$

(c)

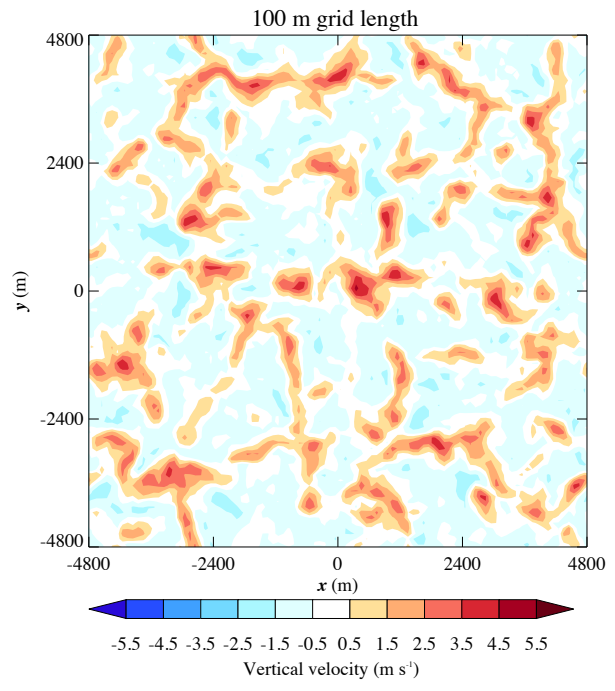

(b)

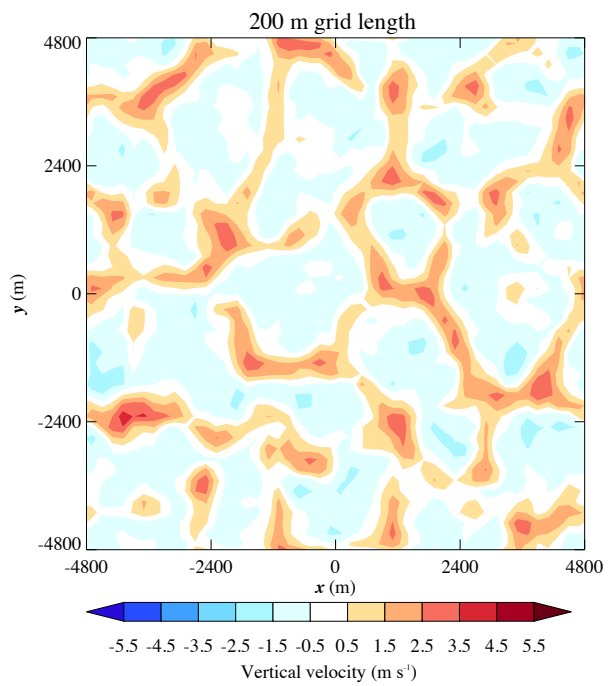

(d)

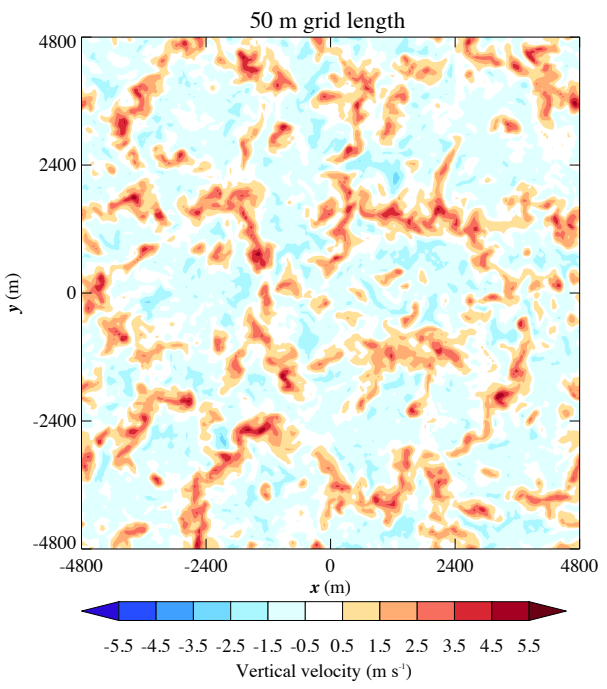

Fig. 2 Horizontal cross-sections of vertical velocity at height $500 \mathrm{~m}$ at time 3.5 hours for horizontal grid lengths of: (a) $400 \mathrm{~m}$, (b) $200 \mathrm{~m}$, (c) $100 \mathrm{~m}$ and (d) $50 \mathrm{~m}$. Contour interval 1 $\mathrm{ms}^{-1}$. 
(a)

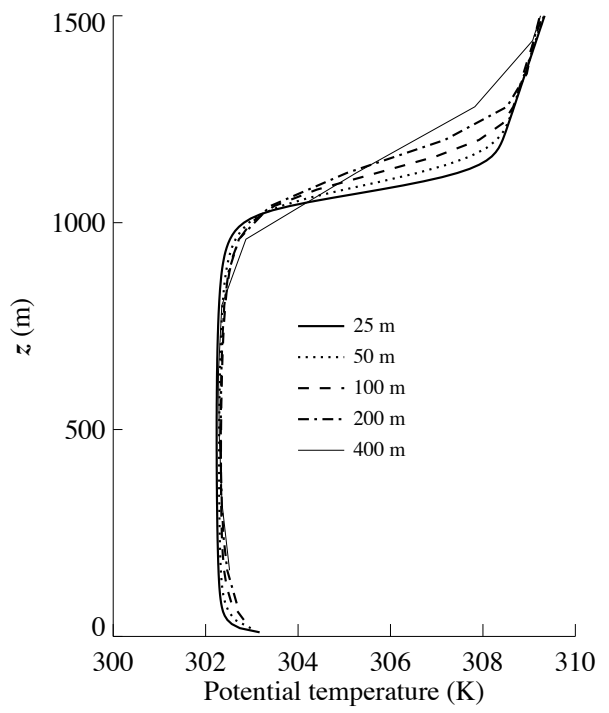

(b)

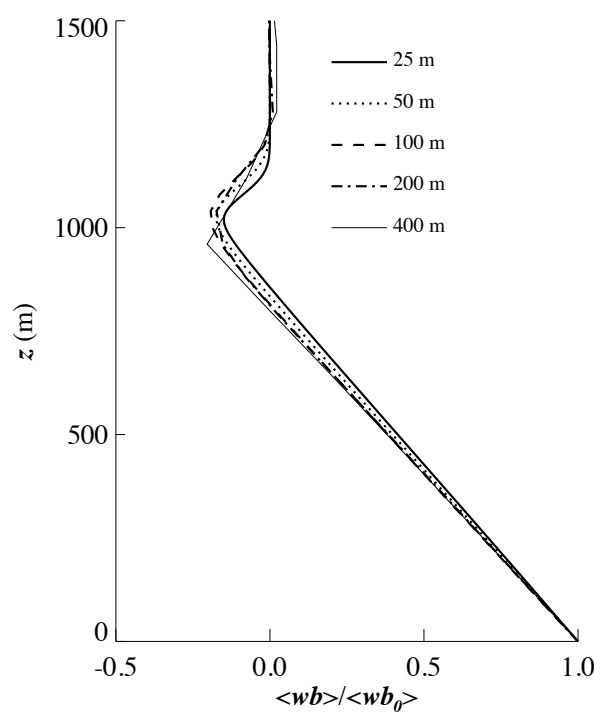

Fig. 3 Vertical profiles of time-area averaged: (a) potential temperature and (b) total (resolved plus subgrid) buoyancy flux profiles $(<w b\rangle$ ), normalised by the surface value, for control. Averaging described in section 2.5. Legend shows different horizontal grid lengths. 


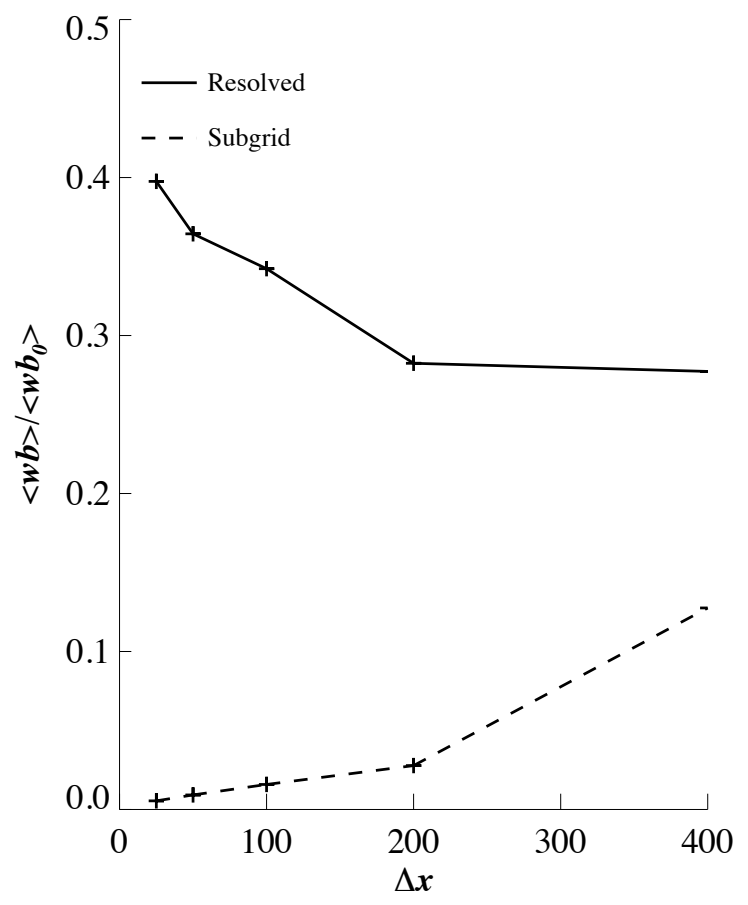

Fig. 4 The variation with grid length of resolved and subgrid buoyancy fluxes at height 500 $\mathrm{m}$, normalised by the surface value, for control simulation. 
(a)

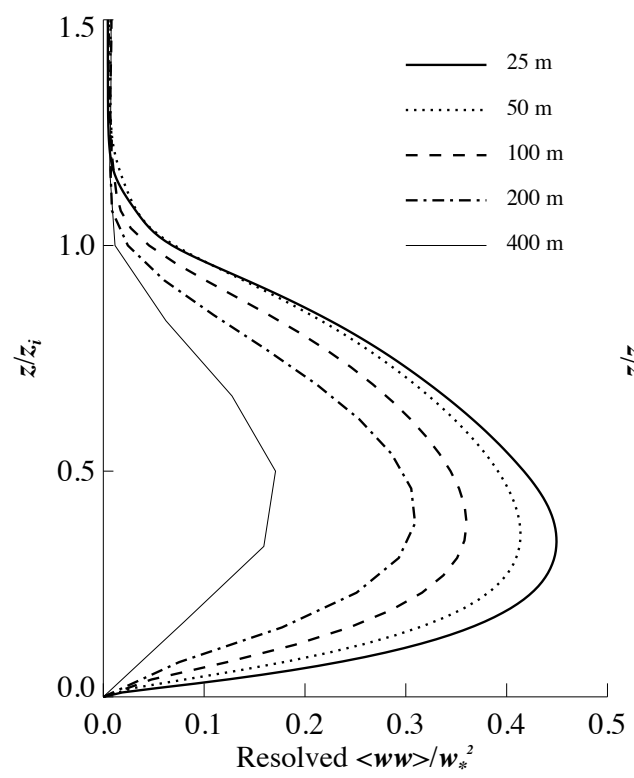

(b)

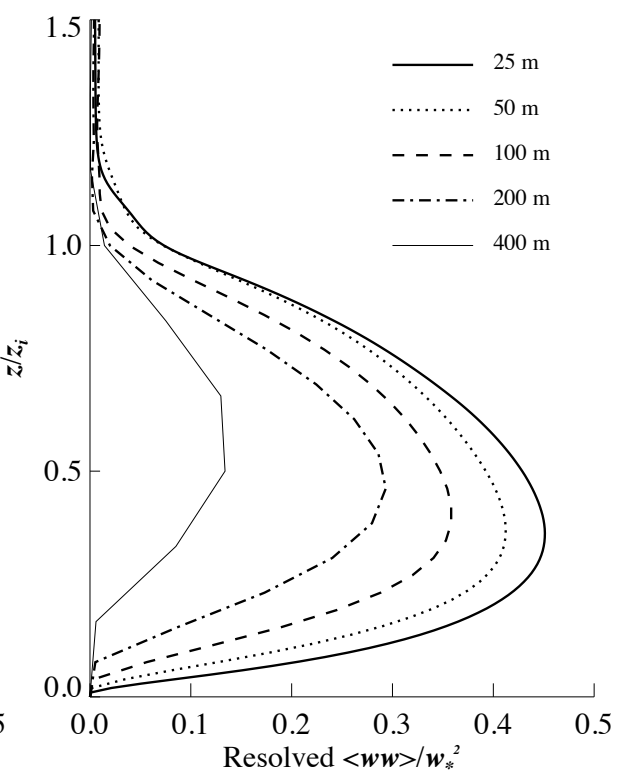

Fig. 5 Resolved time-area averaged vertical velocity variance normalised by $w_{*}^{2}\left(w_{*}\right.$ is the convective velocity scale) plotted against height normalised by inversion depth for: (a) control simulation, (b) TVD on $(u, v, w)$ experiment. Legend shows different horizontal grid lengths. 
(a)

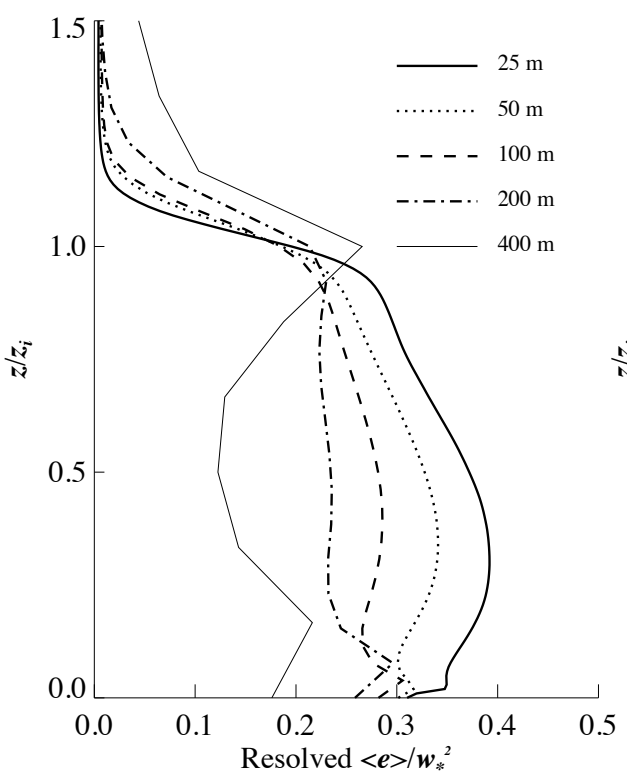

(b)

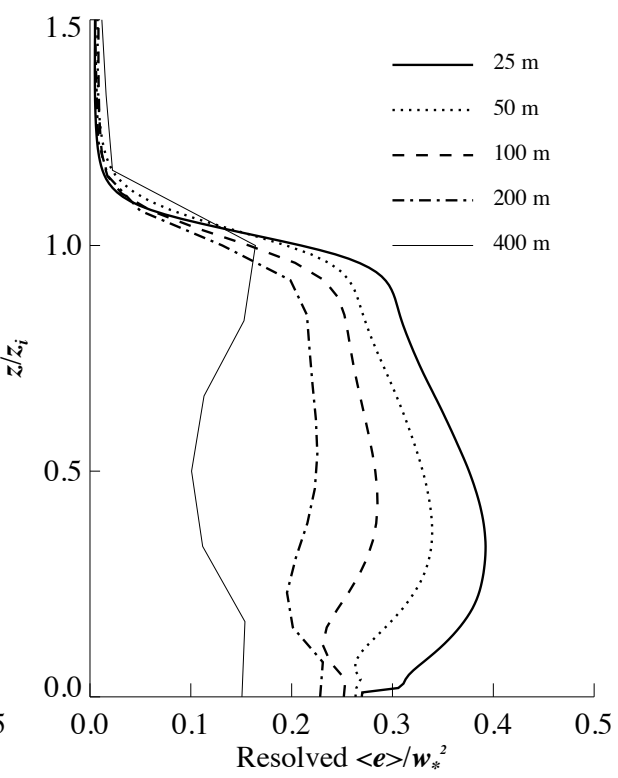

Fig. 6 Resolved time-area averaged turbulent kinetic energy normalised by $w_{*}^{2}\left(w_{*}\right.$ is the convective velocity scale) plotted against height normalised by inversion depth for: (a) control simulation, (b) TVD on $(u, v, w)$ experiment. Legend shows different horizontal grid lengths. 
(a)

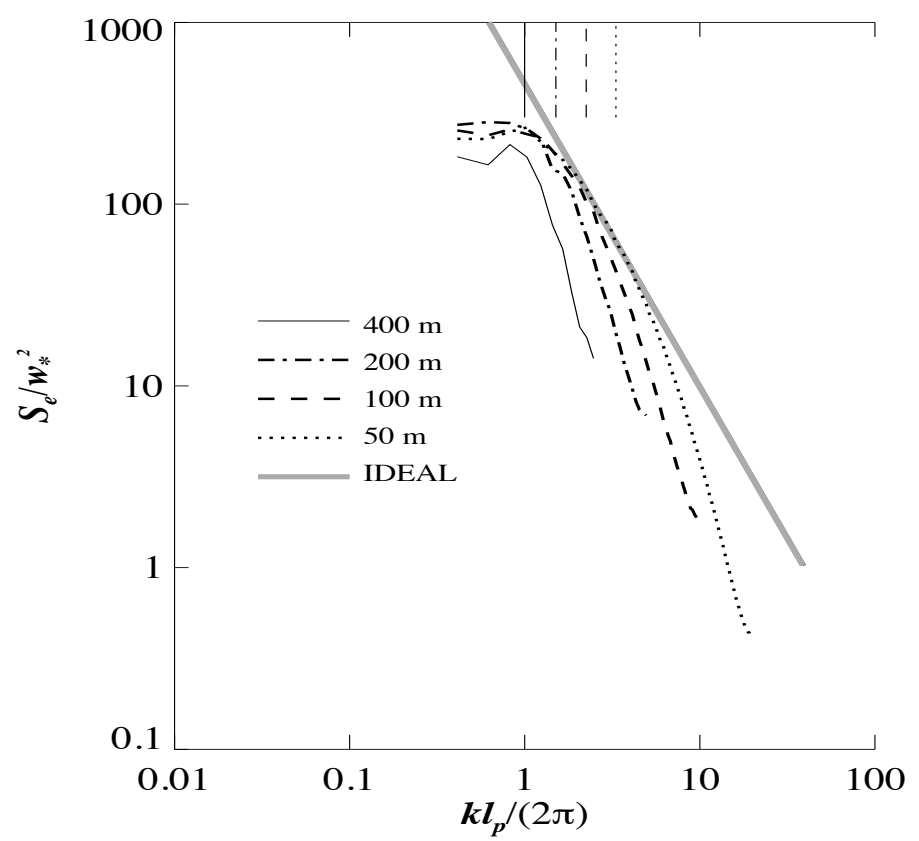

(b)

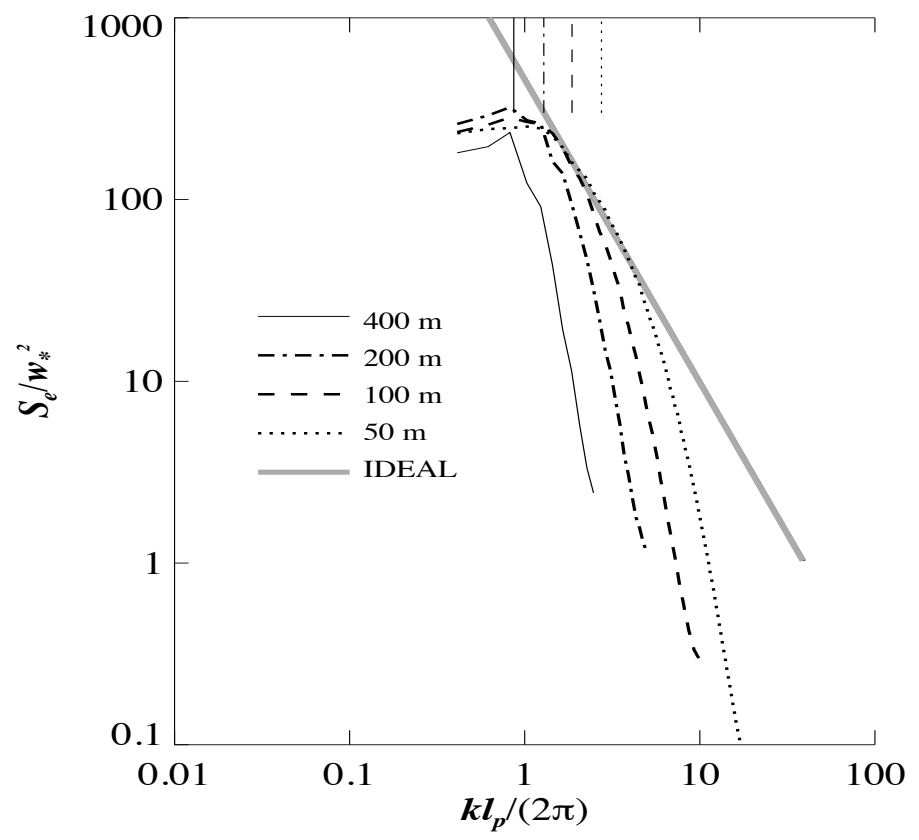

Fig. 7 Time-averaged turbulent kinetic energy spectra $\left(S_{e}\right)$ normalised by $w_{*}^{2}$ plotted against horizontal wavenumber $(k)$ normalised by $\frac{2 \pi}{l_{p}}$ for height $500 \mathrm{~m}$ (middle of boundary layer) and for: (a) control, (b) TVD on $(u, v, w)$. Legend shows different horizontal grid lengths. The location of the dissipation length scales $\left(l_{p} / l_{d}\right)$ for each horizontal grid length plotted as vertical lines at the top of each figure using the same linestyle. The grey line is the ideal $k^{-5 / 3}$ law. For clarity the $25 \mathrm{~m}$ spectrum is omited. 


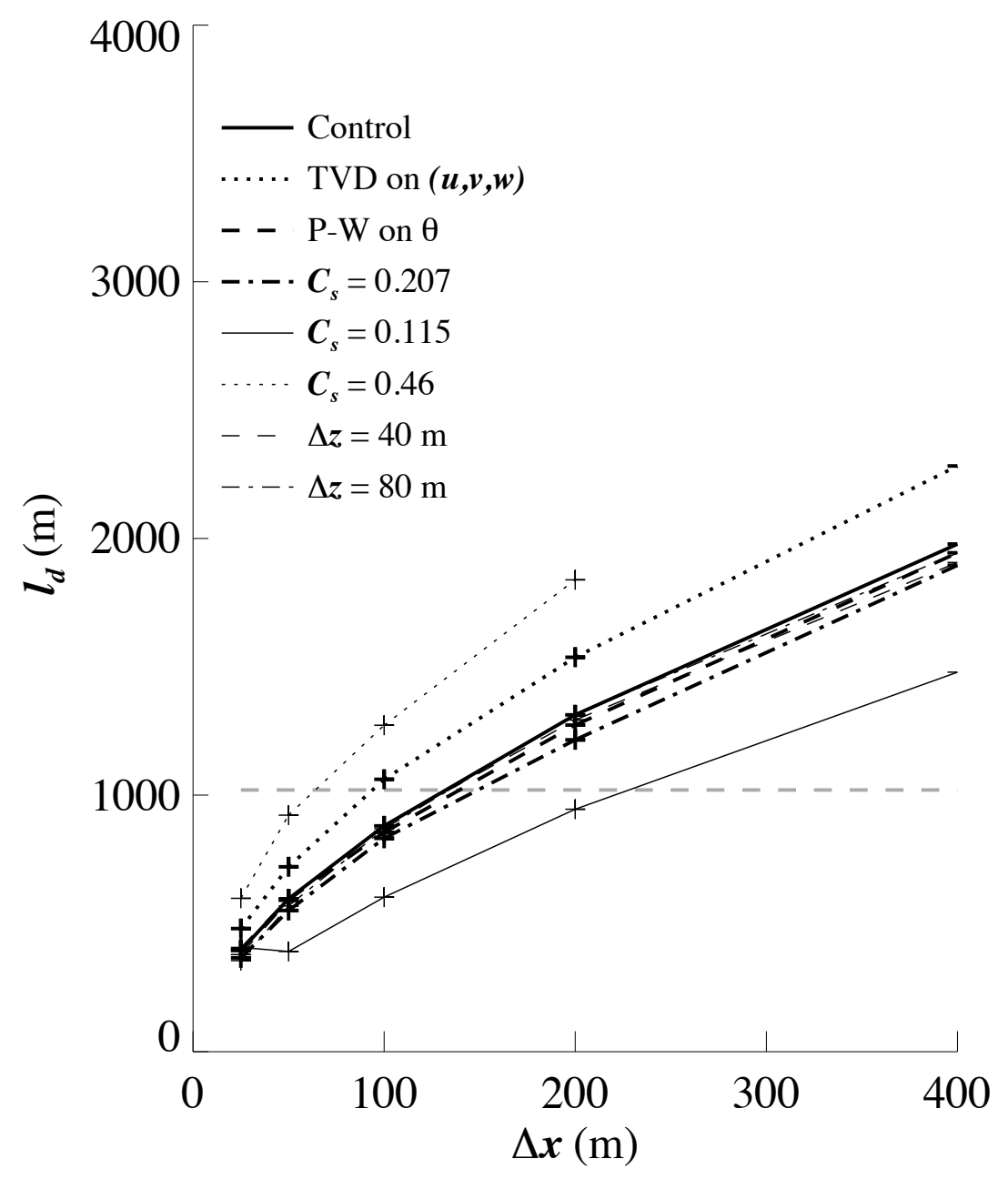

Fig. 8 The dissipation length scale $\left(l_{d}\right)$ plotted against horizontal grid length, evaluated at height $500 \mathrm{~m}$. The inversion depth $\left(z_{i}\right)$ is the grey dashed line. Legend shows the control simulation and other experiments. 
(a)

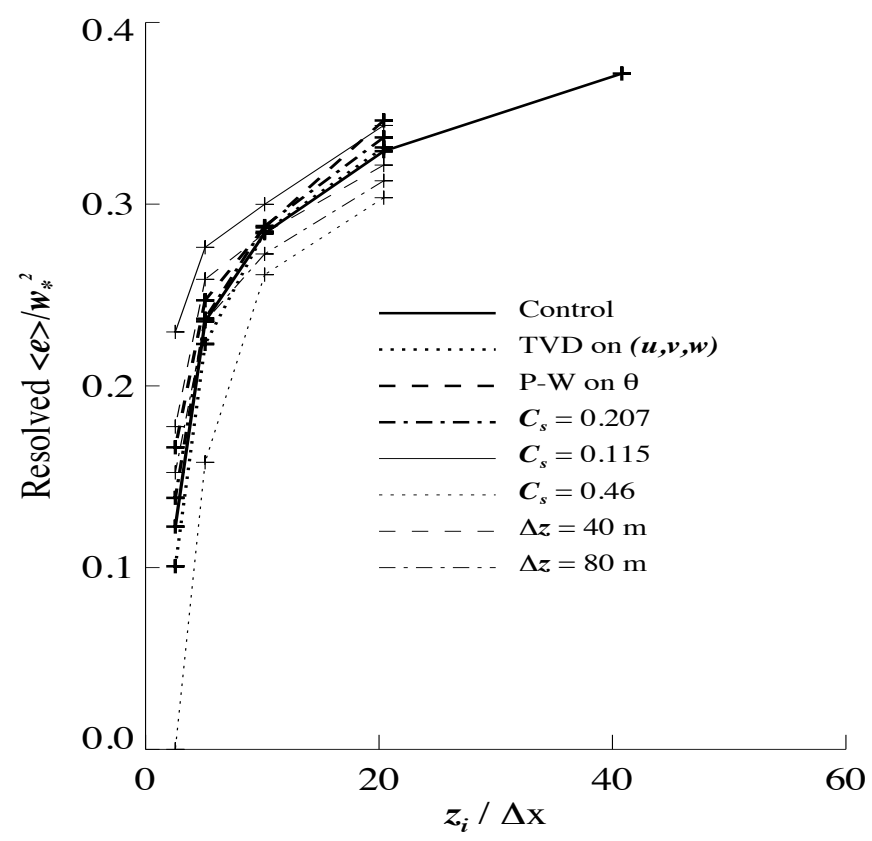

(b)

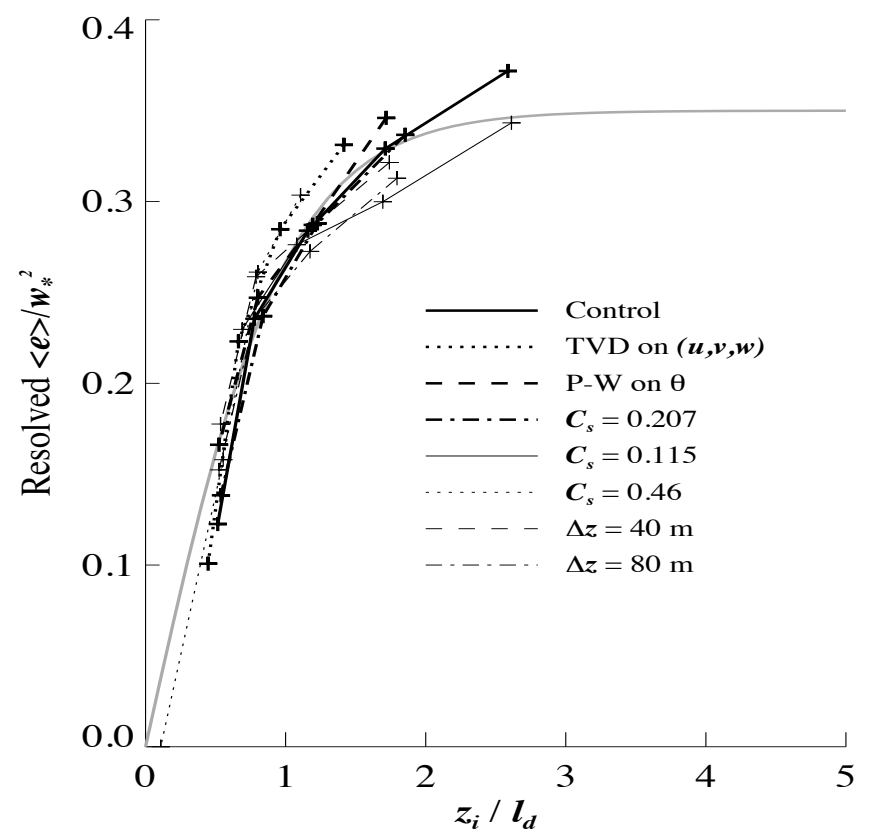

Fig. 9 The resolved time-area averaged turbulent kinetic energy normalised by $w_{*}^{2}$ plotted against (a) $\frac{z_{i}}{\Delta x}$ and (b) $\frac{z_{i}}{l_{d}}$ for each of the grey zone experiments at height $500 \mathrm{~m}$. The grey line is the similarity law in Eq. 7. Legend shows the control simulation and other experiments. 
(a)

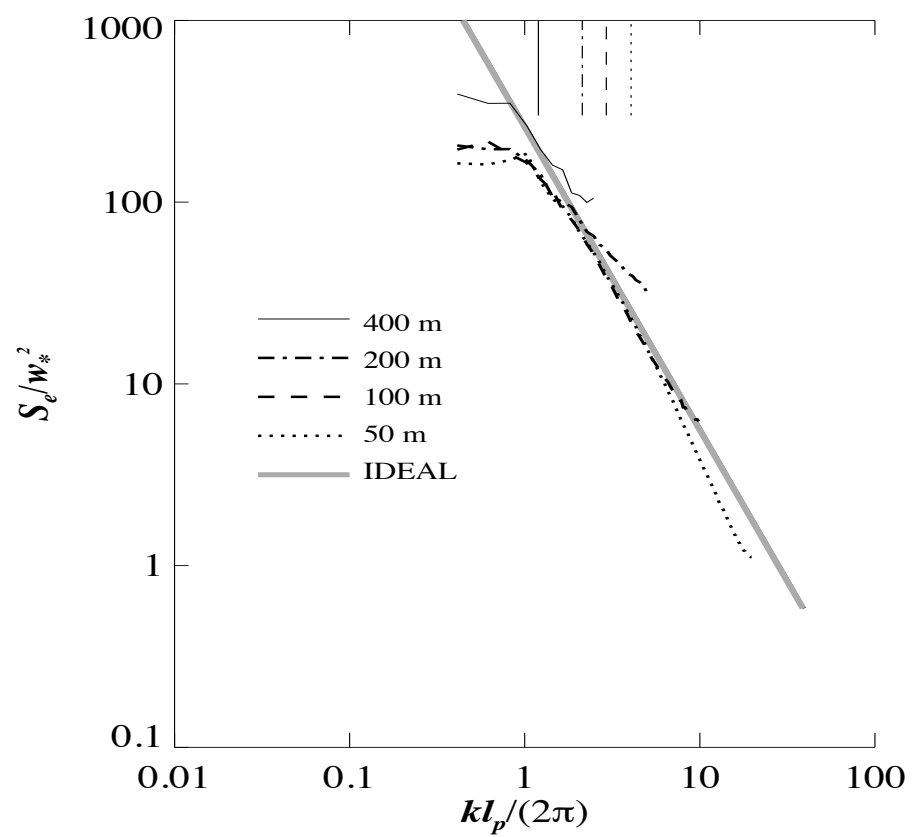

(b)

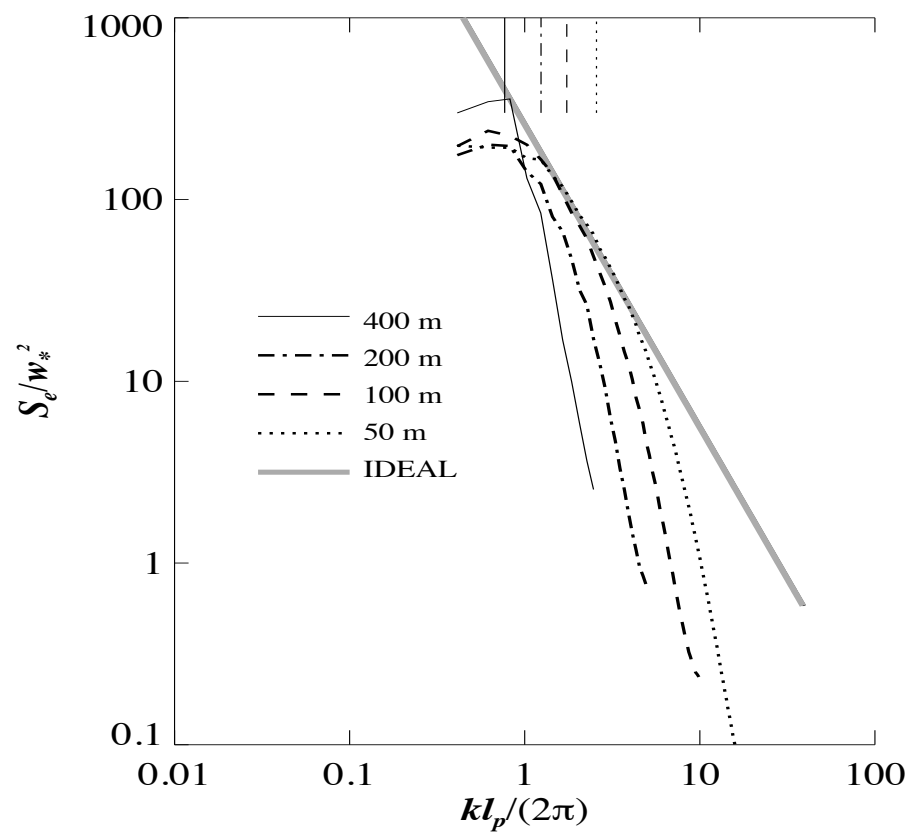

Fig. 10 Time-averaged turbulent kinetic energy spectra $\left(S_{e}\right)$ normalised by $w_{*}^{2}$ plotted against horizontal wavenumber $(k)$ normalised by $\frac{2 \pi}{l_{p}}$ for height $1000 \mathrm{~m}$ (inversion) and for: (a) control, (b) TVD on $(u, v, w)$. Legend shows different horizontal grid lengths. The location of the dissipation length scales $\left(l_{p} / l_{d}\right)$ for each resolution plotted as vertical lines at the top of each figure using the same linestyle. The grey line is the ideal $k^{-5 / 3}$ law. 


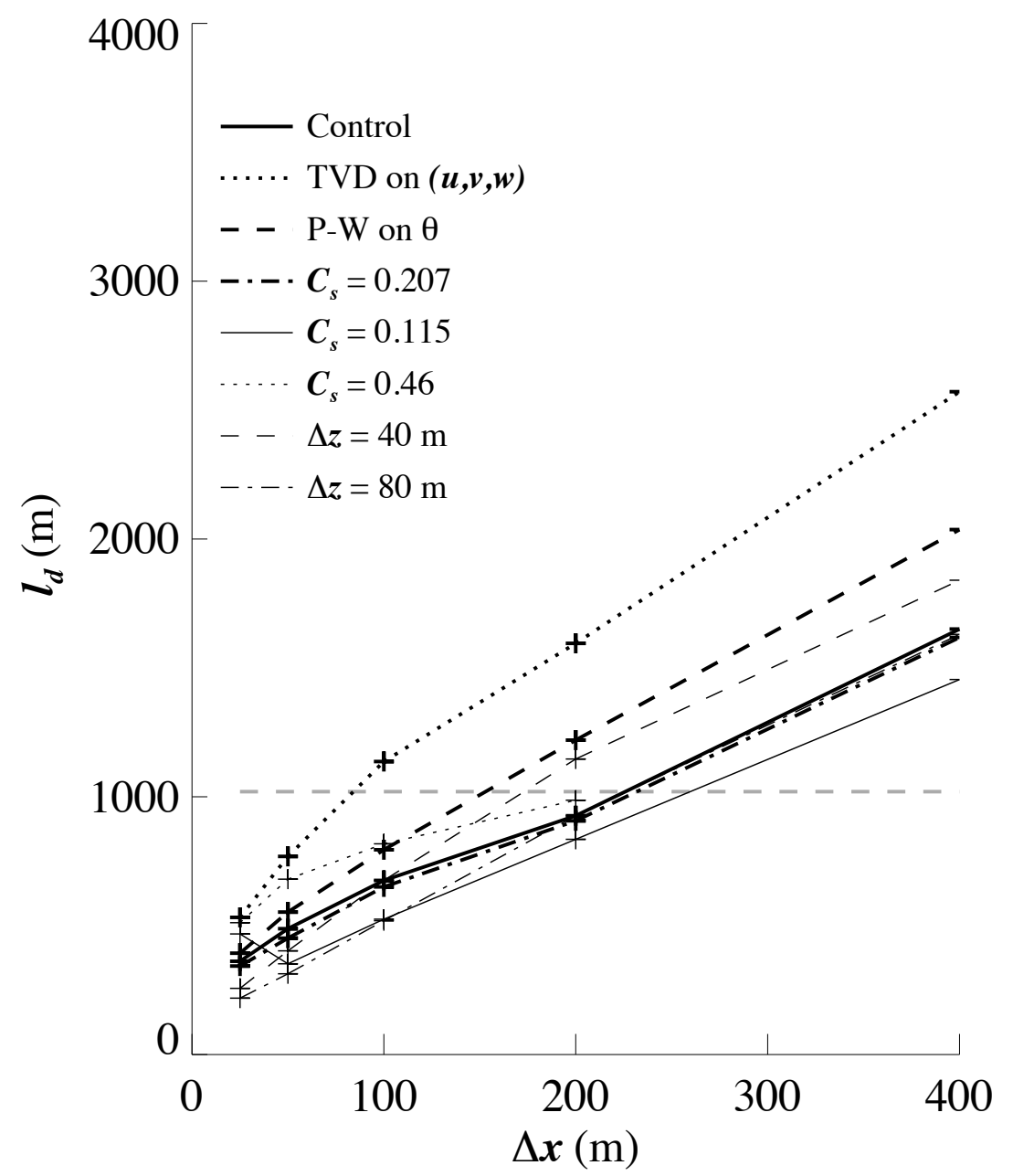

Fig. 11 The dissipation length scale $\left(l_{d}\right)$ plotted against horizontal grid length, evaluated at height $1000 \mathrm{~m}$. The inversion depth $\left(z_{i}\right)$ is the grey dashed line. Legend shows the control simulation and other experiments. 